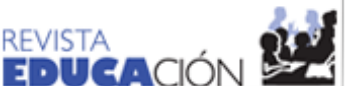

Revista Educación

ISSN: 0379-7082

ISSN: 2215-2644

revedu@gmail.com

Universidad de Costa Rica

Costa Rica

\section{Los Grupos Interactivos en la formación para la sostenibilidad del estudiantado de ingeniería}

Díaz García, Juan Isidro; Chacón-Corzo, María Auxiliadora

Los Grupos Interactivos en la formación para la sostenibilidad del estudiantado de ingeniería

Revista Educación, vol. 45, núm. 2, 2021

Universidad de Costa Rica, Costa Rica

Disponible en: https://www.redalyc.org/articulo.oa?id=44066178027

DOI: https://doi.org/10.15517/revedu.v45i1.43541

\section{(c) $(1) \Theta \Theta$}

Esta obra está bajo una Licencia Creative Commons Atribución-NoComercial-SinDerivar 3.0 Internacional. 
Artículos científicos de experiencia social o didáctica

\section{Los Grupos Interactivos en la formación para la sostenibilidad del estudiantado de ingeniería}

Interactive Groups and Sustainability Training among Engineering Students

Juan Isidro Díaz García

Universidad Nacional Experimental del Táchira,

Venezuela

jidiaz@unet.edu.ve

iD https://orcid.org/0000-0002-2650-793X

Maria Auxiliadora Chacón-Corzo

Universidad de Los Andes Núcleo Táchira, Venezuela

corzomar2@gmail.com

iD https://orcid.org/0000-0002-6725-1256
DOI: https://doi.org/10.15517/revedu.v45i1.43541

Redalyc: https://www.redalyc.org/articulo.oa? $\mathrm{id}=44066178027$

Recepción: 07 Septiembre 2020

Aprobación: 03 Noviembre 2020

\section{RESUMEN:}

El objetivo de este trabajo es presentar los resultados de una experiencia desarrollada en la Universidad Nacional Experimental del Táchira (UNET), Venezuela, mediante la estrategia de Grupos Interactivos (GI). Participaron diez estudiantes de Ingeniería Ambiental, Civil, Mecánica e Industrial, que realizaban sus Proyectos Especiales de Grado (PEG) y respondieron un guion de preguntas para valorar su experiencia como integrantes del GI. El análisis de la información fue de tipo cualitativo e interpretativo, es decir, se examinaron, compararon e interpretaron cada una de las respuestas de los y las informantes en proceso cíclico y recursivo para agrupar y configurar tres categorías: valoración de la participación y aprendizaje en el grupo interactivo, aprendizajes sobre sostenibilidad como profesional de la ingeniería, y relación entre sostenibilidad y trabajo colaborativo. Con base en los resultados, puede afirmarse que los GI les permitieron aprender juntos y comprender su compromiso como personas ingenieras en la protección de los recursos naturales. Por tanto, se considera necesario que el profesorado de las carreras de ingeniería incorpore en sus prácticas los principios de la sostenibilidad, orientados a formar profesionales conscientes de su responsabilidad en el respeto y preservación del planeta y, en este sentido, los GI constituyen una posibilidad para el trabajo en equipo y el aprendizaje compartido de un tema actual y pertinente como la sostenibilidad.

Palabras clave: Grupos interactivos, Sostenibilidad, Carrera de Ingeniería, Desarrollo Sostenible, Educación para el Desarrollo Sostenible.

\section{Abstract:}

The National Experimental University of Táchira (UNET) in Venezuela, conducted an Interactive Group (IG) analysis among ten students majoring in Environmental, Civil, Mechanical and Industrial Engineering participated while they were completing their graduation projects (Special Degree Projects (PEG)). For purposes of the analysis, the students were asked to respond to various scripted questions to assess their experience as a member of the IG. The information analysis was qualitative and interpretive, that is, each informant response was examined, compared and interpreted in a cyclical and recursive process and were grouped together according to three categories: Participation and Learning Assessment from participating in the Interactive Group , Learning about Sustainability as an Engineering Major and the Relationship between Sustainability and Collaborative Group Work. Based on the results, it can be affirmed that the IG analysis fostered shared learning and understand of the group's commitment as engineers to protection our natural resources. College Engineering Instructors must aim at incorporating the principles of sustainability in their lessons in order so that the next generation of engineers will be imbued with a sense of awareness and respect for conservation of the planet. As a result, SIG proved to be an opportunity to leverage teamwork and shared learning regarding this current and relevant topic.

KEYWORDS: Interactive Groups, Sustainability, Engineering Major, Sustainable Development, Sustainability Education. 


\section{INTRODUCCIÓN}

La declaración de la Década de la Educación para el Desarrollo Sostenible (2005-2014) de parte de la UNESCO (2005), con el fin de construir un mundo con acceso igualitario a una educación coherente con un futuro sostenible, permitió impulsar la inclusión del concepto de sostenibilidad en las universidades del mundo. Personas investigadoras como Aznar y Ull (2009) proponen que todo el estudiantado debería desarrollar una comprensión de la sostenibilidad, para que en el futuro considere esta perspectiva en sus actividades profesionales. Otras como Tobón (2005) afirman que se deben reforzar las competencias ambientales de las personas profesionales formadas en las casas de educación superior. Esta introducción de la sostenibilidad en los estudios universitarios se ha definido como sostenibilización del currículo y se orienta hacia un enfoque transversal, para que cada disciplina, desde su área específica, aporte conocimientos, métodos o soluciones para el desarrollo sostenible. Lograr la sostenibilización curricular implica, de acuerdo con lo estipulado por la asociación sin ánimo de lucro llamada Conferencia de Rectores de la Universidades Españolas (CRUE, 2012), "revisar los currículos desde la perspectiva del Desarrollo Sostenible y asegurar una docencia coherente con los principios de Desarrollo Sostenible" (p. 7).

En el caso de las carreras de ingeniería, de todos los Objetivos de Desarrollo Sostenible (ODS) (Naciones Unidas, 2016), se pueden aplicar directamente al objetivo número 4: Garantizar una educación de calidad inclusiva y equitativa, y promover las oportunidades de aprendizaje permanente para todos y todas; y el número 12: Garantizar las pautas de consumo y de producción sostenibles. Estos dos objetivos deben relacionarse desde el punto de vista de la formación de profesionales que van a representar el cambio de paradigma desde la economía lineal a la economía circular. De acuerdo con Murray, Skenne y Haynes (2015), el sistema económico lineal de "tomar-hacer-consumir-desechar" (p. 5), debe avanzar hacia un modelo de economía circular (CE), donde los materiales y productos se reutilizan durante el mayor tiempo posible en sistemas de circuito cerrado. Este hecho permitiría utilizar a la economía circular como una estrategia instrumental para la implementación del desarrollo sostenible, al lograr que los residuos de unas personas se conviertan en materias primas para otras, de manera que los productos, componentes y recursos en general, mantengan su utilidad y valor en todo momento de la producción, o lo que es lo mismo, alcanzar el denominado residuo cero (The Ellen MacArthur Foundation, 2017), con el diseño de productos para ser deconstruidos y totalmente reaprovechables para otro proceso de producción posterior a la economía circular.

El propósito de este trabajo es presentar los resultados de una experiencia desarrollada en la Universidad Nacional Experimental del Táchira (en adelante, UNET), Venezuela, mediante la organización de Grupos Interactivos (GI) como una estrategia dirigida al alumnado de las carreras de Ingeniería Ambiental, Civil, Mecánica e Industrial, cuyo objetivo fue analizar los aprendizajes y experiencias sobre sostenibilidad a través de los GI implementados durante la realización de los Proyectos Especiales de Grado (PEG) asociados a temas de Economía Circular aplicada a la ingeniería.

\section{EsTUDIOS PREVIOS}

La relevancia de los GI, como estrategia para desarrollar el aprendizaje dialógico e impulsar el trabajo colaborativo, se manifiesta en la diversidad de investigaciones que se vienen realizando; por ejemplo, Prados y Aguilera (2013) presentan que, desde el año 2007, la Universidad de Sevilla ha promovido la formación de voluntariado universitario que colabora en grupos interactivos en institutos de educación secundaria que se han constituido como comunidades de aprendizaje, dicha actividad está orientada a estudiantes de cualquier carrera con interés por la educación y la transformación social; el estudiantado universitario participante manifiesta que la actividad le aporta en su formación profesional y en su desarrollo personal.

Por su parte, Flecha, Racionero, Tintoré y Arbós (2014) recogen las actuaciones educativas de éxito en la universidad, las cuales permiten el aumento del rendimiento académico y promueven las relaciones sociales, 
entre ellas destacan los Grupos de Lectura (Reading Groups) que están conformados por estudiantes de posgrado y profesorado. En estos grupos se promueve el diálogo igualitario, al generar debates acerca de problemáticas actuales y las líneas de investigación vigentes del grupo.

También, Gómez y Aguilera (2015) presentan un detallado cuestionario sobre la valoración acerca de los aspectos positivos que tiene el estudiantado participante en grupos interactivos. Los resultados obtenidos presentan una valoración general muy alta para los aspectos positivos de la experiencia, donde la población estudiantil está bastante satisfecha con haber participado voluntariamente en la experiencia.

Otras investigaciones se han orientado a comprobar si las percepciones del estudiantado universitario, participante en los grupos interactivos, se corresponden con lo referido en la literatura. Al respecto, Gómez-del Castillo, Aguilera-Jiménez y Prados-Gallardos (2016) afirman que, efectivamente al trabajar en grupos interactivos, los y las estudiantes manifiestan que es una experiencia positiva al promover "aumento de la motivación por aprender, aceptación de las diferencias, favorecimiento del diálogo, ayuda entre iguales" (p.292). Ponce (2017) llevó a cabo un estudio bajo la modalidad de investigación acción dirigida a estudiantes de nivel universitario en México, cuyos resultados muestran que la implementación de grupos interactivos incrementó el desempeño académico, el trabajo en equipo y la autorregulación, aunque se debe insistir en mejorar la dimensión social.

Finalmente, Tuzun (2020) presenta un ejemplo del uso de grupos interactivos para proyectos de tesis en la universidad, estos relacionados con la sostenibilidad. Su investigación sobre la enseñanza interactiva de la sostenibilidad para personas ingenieras químicas de la Universidad de Cambridge en el Reino Unido, utiliza la metodología del aprendizaje basado en problemas y el desarrollo de la metacognición, propuesta por Downing (2009), en grupos interactivos, donde el aprendizaje del estudiantado se logra mediante estrategias que incluyen observaciones de los y las estudiantes en grupos, entrevistas grupales, sesiones con la persona tutora, asesora industrial, y el uso de paquetes de software para el diseño y optimización de procesos. Con estas actividades se logra la reflexión, el seguimiento sistemático y la utilización constructiva del conocimiento acumulado en los niveles previos de estudio académico. Los resultados obtenidos reflejan un aumento de la satisfacción del estudiantado que participó en los grupos interactivos.

\section{Referentes Teóricos}

\section{Sostenibilidad y Economía Circular (EC)}

En principio, el Informe Brundtland, desarrollado por la Organización de las Naciones Unidas [ONU], (1987), define la sostenibilidad como "el desarrollo que satisface las necesidades del presente sin comprometer la capacidad de las futuras generaciones, garantizando el equilibrio entre el crecimiento económico, el cuidado del medio ambiente y el bienestar social" (p.103). El concepto de sostenibilidad es muy dinámico. De acuerdo con Sinakou, Boeve, Goossens, y Van Petegem (2018), puede ser entendido de muchas maneras, esto depende de la visión de las personas investigadoras, pues cada una puede definirlo desde su campo de estudio. La pedagogía de la sostenibilidad ha sido planteada como un modelo práctico y adaptable para enseñar sostenibilidad (Burns, Sybil, y Spalding, 2019), esta se enfoca en sistemas interconectados, co-crea contenido; cuestiona críticamente las normas dominantes e incorpora diversas perspectivas; utiliza procesos activos, participativos, experienciales y relacionales, e integra el aprendizaje interdisciplinario con múltiples perspectivas, además es activo y comprometido.

Para efectos de este trabajo, la sostenibilidad es un concepto que incluye la protección de los recursos naturales del planeta y la opción de las futuras generaciones de disfrutar de un ambiente limpio, con una economía viable a largo plazo y dentro de una sociedad más equitativa en la distribución de la riqueza obtenida a partir de los recursos, esto mediante el uso de procesos de ingeniería sostenible. Al respecto, Antunes 
y Gadotti (2005) expresan que "la sostenibilidad tiene que ver con la relación que tenemos con nosotros mismos, con los demás y con la naturaleza” (p. 143), definición que podría ser aplicada en el aula de clase para involucrar al alumnado de ingeniería con los problemas asociados al desarrollo sostenible y el futuro del planeta.

Por otra parte, comprender la EC implica empezar por el concepto propuesto por McDonough y Braungart (2005), denominado Cradle to Cradle, o Cuna a Cuna, que consiste en una manera diferente de idear, diseñar y producir, de forma que los elementos que componen los productos puedan ser 100\% reutilizados o reciclados. Para estas personas autoras, existen nutrientes biológicos y nutrientes tecnológicos que pueden ser asimilados por la naturaleza en ciclos sin fin si se diseñan desde un principio para ello.

Otras visiones como la del plan de acción de la UE define la EC como "aquella en la cual el valor de los productos, los materiales y los recursos se mantenga en la economía durante el mayor tiempo posible, y en la que se reduzca al mínimo la generación de residuos" (Comisión Europea, 2015, p. 2). Esto supone un cambio radical del concepto tradicional del sistema de producción, porque involucra cambios, tanto en el diseño como en el desarrollo de los productos, que toman en cuenta la economía y la protección al ambiente.

\section{Ingeniería sostenible}

El modelo de explotación actual de los recursos naturales ha estado orientado por la visión de un mundo donde existe una gran cantidad de fuentes casi infinitas, visión que ha ido cambiando a lo largo de los últimos 20 años, en función de la certeza de la caída en volúmenes y la falta de nuevos yacimientos de la mayoría de minerales y combustibles del Planeta Tierra. La educación de los ingenieros e ingenieras siempre se orientó a aprovechar los recursos, pero en la actualidad se debe reorientar la ingeniería, en todas sus variantes, al correcto uso y máximo aprovechamiento de las capacidades de cada material que se vea involucrado en los procesos industriales. Una posible manera de lograrlo es promover el cuidado de los recursos naturales en los principales agentes transformadores del entorno, como lo serán los futuros ingenieros e ingenieras. Para ello, se debe orientar la práctica educativa en el terreno de la ingeniería sostenible y, de esta manera, insertarla dentro del currículo de las carreras de ingeniería de forma transversal, lo que se ha denominado como "ingenieros con educación ambiental" de acuerdo con Painter (2003, p.267).

Más recientemente, se utiliza el término educación en ingeniería sostenible, que, de acuerdo con Cristina (2016), es aquella educación destinada a desarrollar la personalidad del ingeniero o la ingeniera en niveles tecnológicos, económicos, sociales, gerenciales y morales, que conduzcan a resultados positivos para las personas graduadas y la sociedad en términos económicos, sociales y ambientales.

Los problemas ambientales surgen a partir de problemas humanos, que, debido a sus consecuencias, derivan en acciones que ponen en riesgo la sobrevivencia del planeta. Por eso, toda propuesta de mejoramiento en educación, en cuanto a la gestión ambiental, debe partir de la toma de conciencia, actitudes y conductas individuales. Por tanto, es fundamental incluir a las y los profesionales de todos los ámbitos de la ingeniería que se han graduado en la tendencia mundial de la conservación y aprovechamiento de recursos.

En algunos países como Colombia, uno de los lineamientos de la Política Nacional de Educación Ambiental, del Ministerio del Medio Ambiente y Ministerio de Educación Nacional (2002), considera que "la universidad debe desarrollar estrategias tendientes a introducir la pedagogía, la didáctica y la investigación en educación ambiental como componentes importantes de los diferentes programas de formación" (p. 97). En este sentido, vale resaltar la necesidad de promover la sostenibilidad mediante el uso de la pedagogía ambiental en universidades, labor que debe ser auspiciada por el Estado a través de sus políticas públicas, e implementada por las autoridades universitarias a través de cursos de desarrollo profesional sobre la inserción de la sostenibilidad en el currículo, para lograr así la actualización del profesorado en dicho tema.

Por otra parte, es necesario incluir objetivos y competencias que promuevan la sostenibilidad en los planes de estudio de las universidades. Los objetivos de cada asignatura deben estar alineados con los planteamientos 
de las Naciones Unidas (2014) de acuerdo con cada tipo de ingeniería, y las competencias deben incluir la perspectiva del desarrollo sostenible en las capacidades a desarrollar. Huntzinger, Hutchins, Gierke, y Sutherland, (2007) presentan tres vías para integrar el desarrollo sostenible en el currículo: (1) ajustar la sostenibilidad mediante la inclusión de cursos separados sobre sostenibilidad, (2) incorporar las cuestiones de la sostenibilidad en los cursos de la disciplina, y (3) integrar el concepto de sostenibilidad como un paradigma de cambio en los cursos de pregrado. Al respecto, existen diversas experiencias de la integración del desarrollo sostenible en el currículum de ingeniería como la realizada por Cheah, Yun, y Sale (2012); Díaz, Molina y Prato (2016), Díaz, Juárez, García, Rosales y Zambrano-Sandoval (2018), que demuestran que es posible utilizar casos de estudio específicos para lograr la comprensión del concepto de sostenibilidad en estudiantes de ingeniería.

\section{Los grupos Interactivos}

El aprendizaje como proceso individual e interno es posible mediante experiencias y diálogos compartidos, siempre que se estimule la participación, se conozcan las intenciones formativas, y se perfilen estrategias potenciadoras de los aprendizajes, como se plantea en el caso de los GI. De acuerdo con Peirats y López (2013) "Los Grupos Interactivos como método didáctico pretenden lograr una educación de todos y para todos a partir de una agrupación heterogénea de los estudiantes” (p.3). Esta estrategia didáctica se enmarca dentro del proyecto de las comunidades de aprendizaje y el aprendizaje dialógico fundamentado en la interacción comunicativa, la participación en condiciones de igualdad, caracterizados por principios de equidad, solidaridad y respeto mutuo (Aubert, Flecha, García, Flecha, y Racionero, 2010), de manera que una de las claves es promover la enseñanza y el aprendizaje a partir del diálogo y el trabajo cooperativo entre grupos heterogéneos.

Esta metodología persigue una educación igualitaria e inclusiva, fundamentada en siete principios: (a) diálogo igualitario, implica la toma de decisiones conjuntas; (b) inteligencia cultural, basada en el conocimiento y consideración del contexto, cobra singular importancia las experiencias y el aprendizaje previo, lo cual implica tener en cuenta las competencias de cada integrantes de la comunidad; (c) transformación, es evidente que ocurren cambios, por tanto, la valoración y la crítica permanente entre integrantes emerge como condición para incorporar las modificaciones necesarias orientadas a lograr estos cambios; (d) una dimensión instrumental del aprendizaje, es decir, todas las actividades que se lleven a cabo deben ser significativas y producir aprendizajes para la vida; (e) creación de sentido, que no es otra cosa que la conexión entre lo que se aprende y la realidad, los procesos de enseñar y aprender deben ser útiles en la cotidianidad; (f) la solidaridad, todas las personas se ayudan entre sí durante el proceso de aprendizaje; por último, (g) igualdad en las diferencias, es fundamental tener el respeto hacia cada integrante, valorar sus capacidades, habilidades y destrezas, de allí la relevancia de crear condiciones donde cada integrante del GI se trate con igualdad y tenga las mismas oportunidades (Aubert et al., 2010).

\section{Los grupos interactivos como estrategia de formación para la sostenibilidad}

El grupo tenía como propósito común el desarrollo de un proceso de producción de aglomerado livianos (mezcla de arcilla + subproducto), en hornos eléctricos con variables de tiempo y temperatura replicables en la industria, y un producto final sostenible con propiedades similares a las producidas por las empresas del sector. Los subproductos utilizados fueron: cáscaras de coco, cacao, piña, plátano y caña de azúcar, recolectadas en las zonas agrícolas cercanas a la Universidad.

En la Figura 1 se presenta el GI en una reunión. Estas sesiones se realizaban los lunes en la mañana, dada la disponibilidad de todas y todos los miembros. Allí se informaba de los adelantos realizados, las acciones y 
necesidades requeridas para continuar con la investigación. Se realizaron 8 reuniones con una duración de 02 horas cada una, en dichas reuniones era frecuente el aporte individual de cada persona, pues permitía la libre expresión de las ideas y pensamientos al grupo.

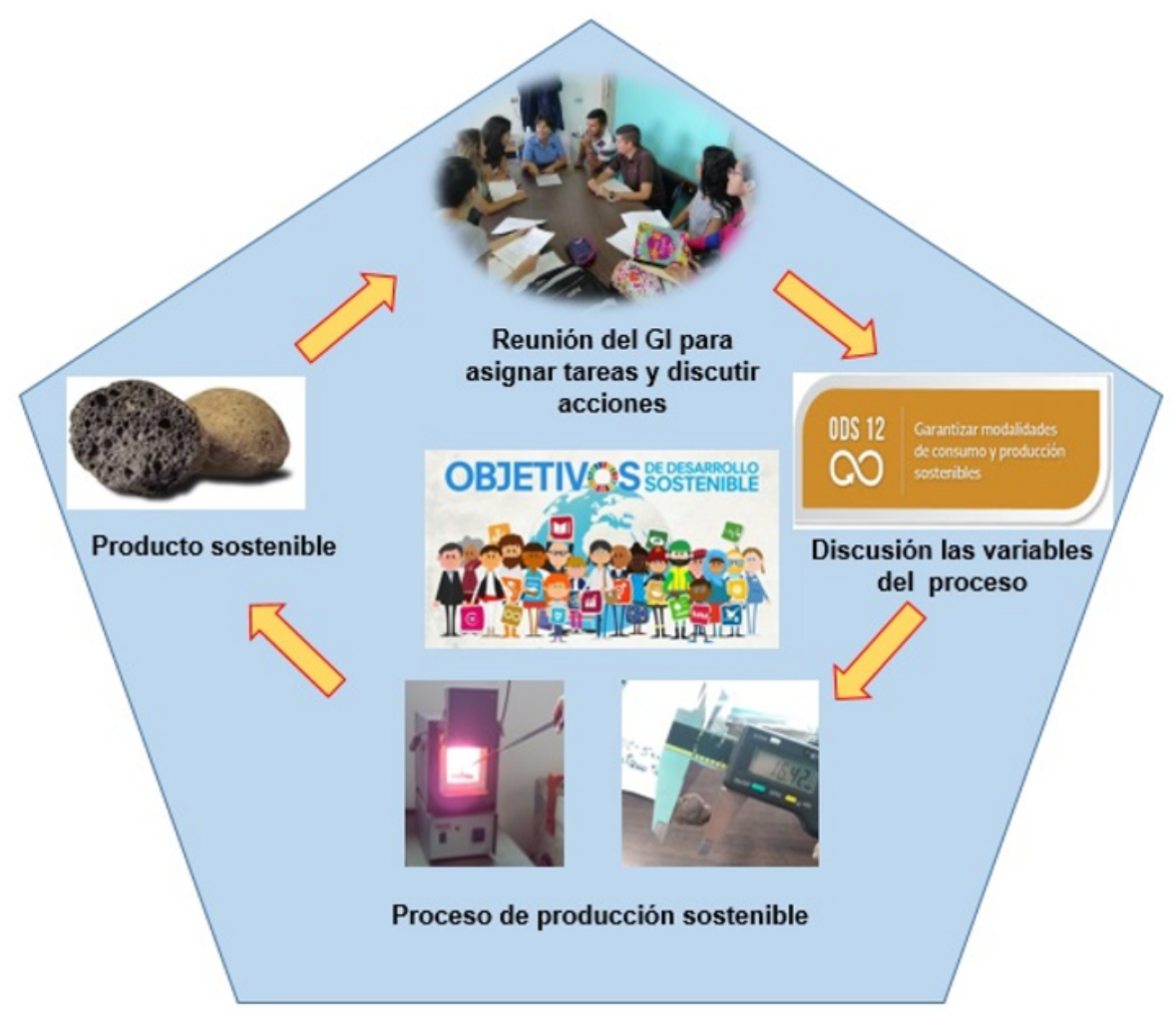

Figura 1. Grupo interactivo

Fuente: Elaboración propia.

Parte del proceso de trabajo consistió en la búsqueda y recolección en las zonas agrícolas de los subproductos a utilizar. Posteriormente, se hacían procesos de secado y quema en las instalaciones del laboratorio, para reducir volúmenes a ser añadidos a la mezcla de arcilla. Allí, el estudiantado de los diferentes equipos, se apoyaban entre sí, independientemente de su carrera de ingeniería.

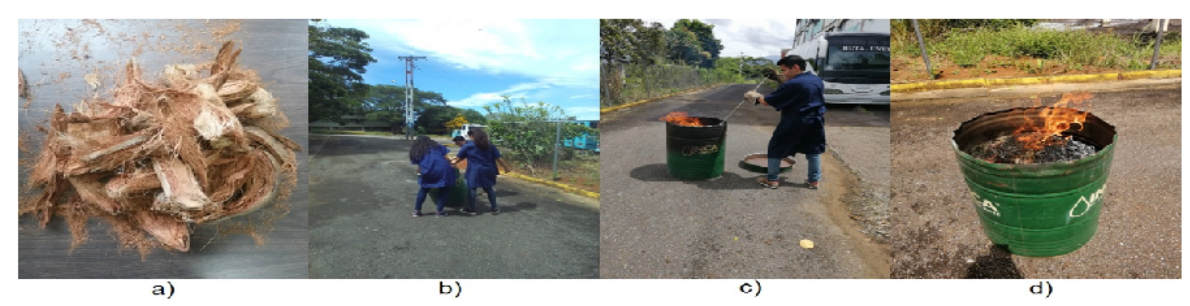

Figura 2 Etapas del proceso de producción sostenible. Fuente: Elaboración propia.

En la Figura 2 se observan las etapas del proceso: (a) cáscara de Coco, subproducto de la zona, (b) proceso de preparación de la calcinación por miembros del grupo interactivo (ingenierías ambiental, civil y mecánica), (c) combustión del subproducto y (d) cenizas de coco para uso sostenible en productos de la construcción. La formación para la sostenibilidad descrita en este artículo estuvo orientada a ambientar el currículo universitario, al tomar como eje central la realización del PEG en grupos interactivos multidisciplinarios, que permitieran la formación de los futuros ingenieros e ingenieras con capacidad para desenvolverse en esta 
sociedad globalizada, como un elemento transversal en la gestión de un conocimiento crítico y pertinente con los procesos formativos sobre la sostenibilidad, desde el punto de vista de su contexto particular y natural.

\section{MÉTODo}

En el desarrollo de esta experiencia se decidió asumir una postura cualitativa, propia de los estudios interpretativos. De acuerdo con Pérez (2000), en este tipo de estudios, se busca el significado y sentido que otorgan las personas a sus conductas. Se organizó el GI con estudiantes del último año de las carreras de ingeniería de la UNET, y en proceso de realización del proyecto especial de grado (PEG). El tema general de todas las tesis estaba relacionado con la sostenibilidad de los subproductos de la región fronteriza Colombovenezolana, pero tratado según las competencias de las diversas ingenierías involucradas.

Para dar respuesta al objetivo planteado, en relación con los aprendizajes y experiencias sobre la sostenibilidad a través de los GI, se decidió indagar sobre la opinión del estudiantado acerca de su participación en el GI, para interpretar el significado que otorgaron a sus acciones orientadas a incentivar procesos de concientización en los futuros ingenieros e ingenieras sobre la importancia de la sostenibilidad.

Ahora bien, para recoger la opinión de las y los participantes sobre sus aprendizajes y experiencias, se elaboró un guion de preguntas abiertas y cerradas que fue enviado por correo electrónico; se empleó la aplicación de Google Forms (Anexo 1). La elaboración del guion se fundamentó en la revisión teórica relacionada con la sostenibilidad y los GI; asimismo, fue valorado por dos personas expertas, cuyas recomendaciones fueron consideradas, previo a enviarlo a quienes participaron.

Una vez recolectada la información, se analizó cada una de las respuestas en un proceso inductivo analítico, para así encontrar similitudes, diferencias y significados (Colás, 2000).

\section{Participantes}

La selección de las personas informantes se realizó con base en un muestreo intencional, definido como una decisión anticipada a partir de la cual se seleccionan informantes según sus conocimientos y experiencias sobre el tópico a indagar (Bonilla-Castro y Rodríguez, 2005). Por su parte, Martínez (2006) señala que "en la muestra intencional se eligen una serie de criterios que se consideran necesarios o altamente convenientes para tener una unidad de análisis con las mayores ventajas para los fines que persigue la investigación” (p. 86). En este sentido, los y las participantes se seleccionaron con base en los siguientes criterios: (a) ser estudiantes de ingeniería del último semestre, (b) estar realizando su PEG, (c) expresión voluntaria a colaborar. El grupo total fue de 10 estudiantes, quienes se dividieron en equipos de dos personas por Proyecto Especial de Grado (PEG), para un total de cinco equipos: un equipo de ingeniería ambiental, un equipo de ingeniería civil, y tres equipos de ingeniería mecánica para cinco PEG diferentes, relacionados con el uso de la economía circular en la ingeniería. Participaban, además de estudiantes, en el rol de persona asesoras: una ingeniera a cargo del laboratorio donde se realizaban los PEG y una persona docente que dirigía de los trabajos de grado, además de diversas personas colaboradoras ocasionales al grupo, relacionadas directamente con el tema de investigación.

\section{AnÁlisis de Resultados}

Producto del análisis e interpretación emergieron tres unidades de análisis definidas por Pérez (2000) como: "núcleos con significado propios" (p.146), conceptuados con base en los datos (Ver Figura 3). 


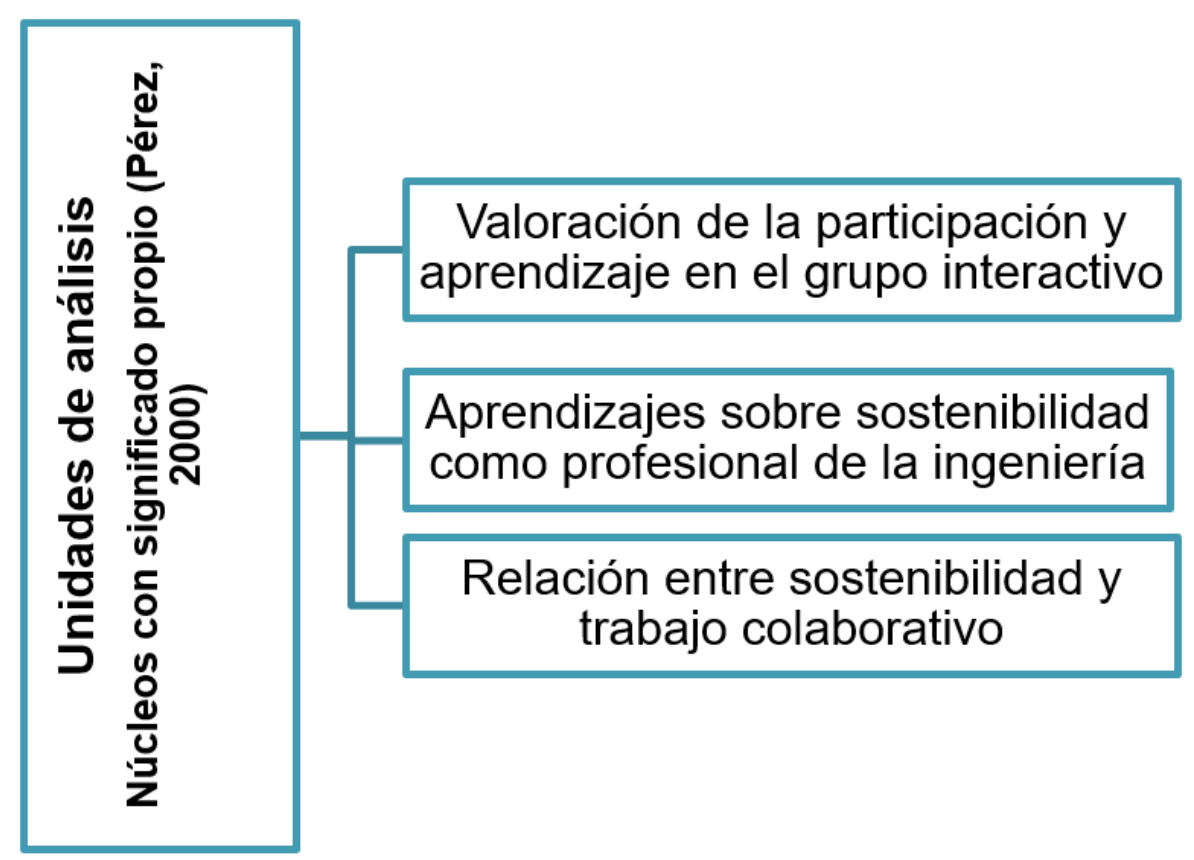

Figura 3 Unidades de análisis emergentes Fuente: Elaboración propia a partir de los datos.

\section{Valoración de la participación y aprendizaje en el grupo interactivo}

Los grupos interactivos se pueden convertir en una poderosa estrategia de aprendizaje, esto cuando se hace hincapié en las potencialidades e interacciones en un contexto de respeto e igualdad, lo cual incide en el aprendizaje de todos y todas sus integrantes. En este caso particular, se potenciaron los intercambios y los aportes a fin de enriquecer el trabajo de cada participante. Podría afirmarse que se ampliaron y complementaron los conocimientos de otras áreas vinculadas a las ingenierías, así lo manifiestan algunas de las personas participantes:

P1: En lo personal, mi experiencia ha sido muy grata, puesto que se genera ideas diferentes lo que es de gran aporte para la realización del PEG y ayuda a despejar dudas que se presentan durante la realización de este. (Participante 1, comunicación personal, 15 de junio 2020).

P4: Los grupos interactivos permiten completar las actividades planteadas a partir del diálogo y la interacción de sus miembros, también se refuerza los valores de solidaridad y compañerismo; se fomenta la inclusión y el aprendizaje cooperativo. (Participante 4, comunicación personal, 17 de junio 2020).

P6: Pude intercambiar información con otros tesistas referente a nuestros proyectos, ayudándonos así a enriquecer los PEG. Siendo de gran ayuda para relacionar y saber la función de mi investigación y las de mis compañeros con otras disciplinas. (Participante 6, comunicación personal, 18 de junio 2020).

En relación con los objetivos comunes y la responsabilidad compartida, entre otros principios del aprendizaje dialógico señalan:

P8: Innovadora, conocer e intercambiar ideas con personas de otras carreras permite ampliar conocimientos y dar solución, o bien, surgir nuevos proyectos apoyados en diversas áreas. (Participante 8, comunicación personal, 19 de junio 2020).

P9: En mi experiencia personal, mejoró mis expectativas de trabajo para la realización del PEG, ya que aprendí a escuchar las opiniones de los demás, comunicarme y ver puntos de vista diferentes a los propios, convivir con personas que me enseñaron un poco más de lo que ya había aprendido a lo largo de la carrera, y recordar lo aprendido en ella en semestres anteriores, y profundizar junto con mi grupo de trabajo lo que deseábamos obtener en la investigación. (Participante 9, comunicación personal, 20 de junio 2020). 
Por otra parte, el estudiantado valoró su aprendizaje al expresar que fue constructivo y fluido. En experiencias basadas en aprendizaje dialógico, es relevante el aprendizaje que resulta de la resolución de problemas en equipo para asumir responsabilidades, compartir conocimientos y aportar soluciones. Al respecto, Ponce (2017), en los resultados de su estudio, señala el incremento de la capacidad de trabajo en equipo, el surgimiento de la autorregulación y la autoestima como capacidades susceptibles de mejorar en los GI; Gómez et al. (2016) afirman que los grupos interactivos permiten al estudiantado una experiencia positiva, al promover la motivación por aprender, la aceptación de las diferencias, el diálogo y la ayuda entre iguales, aunque, por supuesto, exige esforzarse en validar y argumentar sus conocimientos y estrategias, siempre en condiciones de respeto e igualdad (Díez-Palomar, García, Molina y Rué, 2010). En tal sentido, al ser consultados sobre cómo fue el proceso de aprendizaje, las y los estudiantes expresaron:

P1: Un proceso fluido, el cual ayudó a que la realización del PEG se hiciera mucho más fácil y, de cierto modo, más didáctica, lo que generó en mí una motivación distinta. (Participante 1, comunicación personal, 15 de junio 2020).

Asimismo, se muestra el valor que conceden a las aportaciones de sus compañeros y compañeras cuando señalan:

P3: Además de la integración y compartir ideas, fue que los compañeros del área de ingeniería ambiental nos dieron información necesaria, recaudada en su PEG, para nosotros solventar los problemas de diseño del horno rotatorio. (Participante 3, comunicación personal, 17 de junio 2020).

Adicionalmente, desarrollar el trabajo en equipo, apreciar el trabajo interdisciplinario formó parte de los aprendizajes construidos, ello produce sinergia en la consecución de las metas comunes:

P6: Al notar que un mismo tema de trabajo tiene y debe tomar en cuenta todas las ramas que puedan estar allí involucradas, para de esta forma tener un proyecto completo y que cumpla con todas las necesidades. (Participante 6, comunicación personal, 18 de junio 2020).

P7: Al principio, uno se sobresatura porque cree que debe ayudar y hacer de todo. Pero luego va comprendiendo que cada quien tiene su función, y por eso cada quien desarrolla su especialidad en lo que es más bueno, para que el trabajo no se haga tan pesado y se enfatiza en el proceso de investigación llevado a cabo por cada uno, a fin de desarrollar cada una de las etapas exigidas para culminar con el trabajo encomendado. (Participante 7, comunicación personal, 19 de junio 2020).

Derivado del comentario anterior, se evidencia cómo, durante el proceso, las y los participantes descubrieron la necesidad de sus aportes, pero también la capacidad de comprensión de una tarea que es colectiva y no individual. Lo anterior en una suerte de concienciación de la importancia de trabajar en conjunto para alcanzar los objetivos comunes: aprender y culminar con éxito el PEG.

La investigación encuentra un lugar preponderante en el aprendizaje de las personas participantes, quienes la consideran una actividad permanente durante todo el proceso:

P2: El proceso inicial fue netamente de investigación, toda la referencia bibliográfica fue facilitada por el tutor, los análisis y conclusiones fueron discutidos previamente antes de comenzar con la etapa de desarrollo (cálculos, análisis y diseño). Posteriormente, todos los resultados fueron evaluados y discutidos junto con el tutor (Participante 2, comunicación personal, 15 de junio 2020).

P4: En la investigación se implementó el uso de lodos industriales, desperdicios que quedan del proceso, como lo es los lodos rojos. Estos residuos no son sometidos a ningún tipo de tratamiento posterior a su producción, y se aprovecharon para la elaboración de agregados livianos, que posean las características necesarias para ser implementados en la producción de concreto liviano estructural (Participante 4, comunicación personal, 17 de junio 2020).

Una de las personas participantes expresa que, si bien se trató de un proceso que exigió esfuerzo y estudio permanente, fue productivo y significativo:

P9: Durante este tiempo, el proceso fue un poco fuerte, incursionar en un tema extenso, como lo es la sostenibilidad, fue de gran ayuda para completar mis estudios adquiridos durante la carrera. Obtener los antecedentes y leer estudios anteriores fueron de gran ayuda para la realización. Luego, a medida que se fue desarrollando el proyecto, se fue tornando más sencillo y entendible. Ya luego de haber logrado los objetivos, tener una respuesta a cada uno de ellos fue satisfactorio. Saber todo 
lo que aprendimos en este proyecto, como se dice, no tiene precio, esto ayuda a incursionar la vida como ingeniero y aplicar sostenibilidad de mejor modo (Participante 9, comunicación personal, 20 de junio 2020).

Otro aspecto fundamental en el trabajo en grupos interactivos es aprender y compartir conocimientos, procedimientos, y empoderar a quienes participan en experiencias que desarrollen el respeto por las otras personas y la capacidad de trabajar en equipo. En relación a esto, afirmaron:

P9: De ellos aprendí que cada opinión es un grano de arena para realizar cualquier proyecto, que tener diferentes puntos de vista hace ver las cosas de muchas maneras y ver diferentes modos de desarrollar un proceso, un método, un sistema, y de ahí desarrollar de mejor manera lo que se quiera realizar (Participante 9, comunicación personal, 20 de junio 2020).

P7: Que, aunque haya días agotadores, hay días donde la satisfacción de que el éxito sea para todos nos enorgullece de lo que hacemos. Aprendí a lidiar con distintos temperamentos y dejar de pensar solo en mí mismo (Participante 7, comunicación personal, 19 de junio 2020).

En el gráfico presentado en la Figura 4, se destacan los aprendizajes, así: elaborar y utilizar mecanismos de pensamiento para la resolución de problemas; desarrollar procesos de aprendizaje cooperativo, e interactuar con sus compañeros y compañeras para conseguir objetivos comunes, abarca el $80 \%$ de las respuestas. Mientras que desarrollar operaciones cognitivas elementales, tales como la memoria, la observación, el análisis y la síntesis, representa el 60\%; seguido del $70 \%$ en relación con utilizar en el grupo interactivo todas las interacciones necesarias para afrontar exitosamente el PEG en la actual sociedad de la información, y un 30\% de respuestas están vinculadas aprender las reglas prácticas para la ejecución de un dominio de tareas.

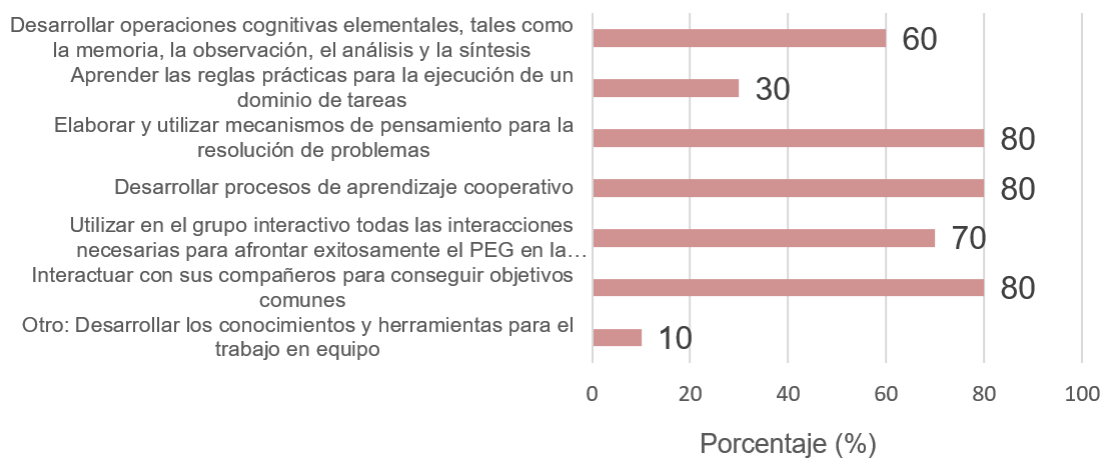

Figura 4. Aprendizajes alcanzados mediante la experiencia de trabajo como GI durante realización del PEG Fuente: Elaboración propia.

Otro aspecto relevante es la visión de las personas participantes en cuanto a las ventajas y desventajas de trabajar en GI (ver Figura 5). De las ventajas destaca el incremento del aprendizaje con el 80\%; le sigue el 70\% de las respuestas vinculadas a compartir aprendizajes. La distribución del tiempo y mejora de la autoestima representan el 60\%, de modo que se deduce que la experiencia de integrar grupos interactivos, para diseñar y ejecutar el PEG, puede considerarse una estrategia adecuada para lograr aprendizajes, no solo vinculados con la profesión, sino para el desarrollo de competencias profesionales que capaciten para el trabajo en equipo, la solidaridad y el respeto por las otras personas, las cuales son necesarias en la sociedad actual. 


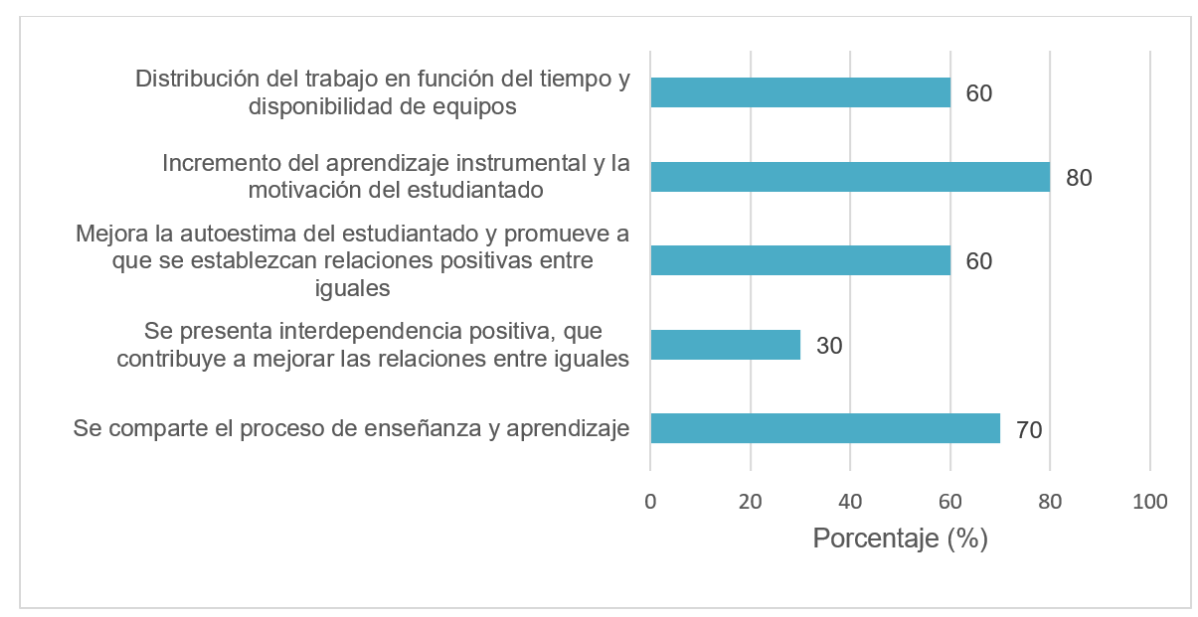

Figura 5. Ventajas del trabajo cooperativo durante realización del PEG Fuente: Elaboración propia.

Asimismo, se evidencian en la Figura 6 las desventajas que pudieran derivarse del trabajo en grupos interactivos, entre las cuales se subrayan: la necesidad de una buena coordinación para trabajar en equipo y la necesidad de mucho tiempo para preparar las actividades, lo cual representa el $70 \%$. Otro aspecto que resulta desventajoso es la falta de práctica para trabajar cooperativamente, esto con el $20 \%$. Le siguen las dificultades para ponerse de acuerdo y el desinterés de algunas personas que integran el grupo. De este modo, puede deducirse que trabajar en un GI exige de la comprensión e implicación de cada a participante para alcanzar el éxito, es decir, trabajar cooperativamente exige de respeto y esfuerzo de todas las personas en aras de obtener los mayores beneficios y un aprendizaje significativo (Aubert et al., 2010; Ponce, 2017).

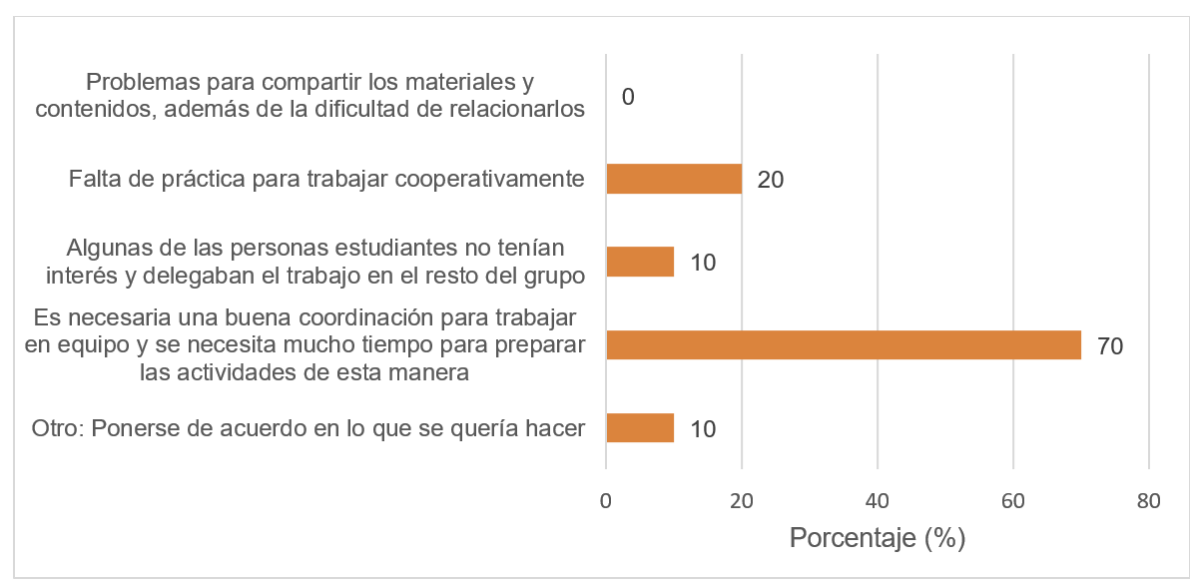

Figura 6 Desventajas del trabajo cooperativo durante la realización del PEG Fuente: Elaboración propia.

\section{Aprendizajes sobre sostenibilidad como profesional de la ingeniería}

Existe una continua reclamación acerca de la carencia de políticas sobre sostenibilidad en las universidades. Antúnez, Gomera, Villamandos (2017) describen que, ante esta realidad, el uso de los GI puede surgir como una alternativa para implicar al profesorado, formar al estudiantado y a futuras personas transformadoras de los procesos industriales en los principios de la sostenibilidad. En cuanto a cuáles serían las competencias requeridas, producto de una consulta realizada por Rieckmann (2012) a diversos expertos sobre las competencias necesarias para la Educación para el Desarrollo Sostenible (EDS), se obtuvieron 19 
competencias con gran relevancia. Para este trabajo son de interés: (a) competencia de pensamiento crítico, que supone la capacidad de analizar los problemas de desarrollo sostenible y (b) pensamiento sistémico y manejo de la complejidad, que implica la habilidad para analizar las interrelaciones que se dan entre las diferentes variables dentro de un sistema y entre sistemas. En tal sentido, al consultar sobre si en su desempeño profesional en ingeniería puede contribuir a la sostenibilidad, los y las estudiantes comentaron:

P10: Sí, nuestro trabajo es crear e innovar proyectos que favorezcan a la humanidad. Por lo que debemos contemplar la sostenibilidad desde el primer paso en cualquier proyecto que se plantee (Participante 10, comunicación personal, 20 de junio 2020).

P6: Sí, es una de las razones por las que elegí esta carrera universitaria, ya que me ha proporcionado parte del conocimiento relacionado a cómo tener procesos más viables. Me agrada y espero formar parte de más proyectos donde el factor ambiental sea tan importante como el producto final. (Participante 6, comunicación personal, 18 de junio 2020).

En general, los miembros del GI plantean una relación directa entre la sostenibilidad y la carrera de ingeniería:

P5: Los conceptos de ingeniería están en congruencia con los esfuerzos para realizar proyectos sostenibles, simplemente debe emplearse el enfoque adecuado (Participante 5, comunicación personal, 18 de junio 2020).

P9: En mi desempeño como ingeniero, espero aplicar mis conocimientos adquiridos, para así lograr la contribución a los sistemas sostenibles en cualquier proceso (Participante 9, comunicación personal, 20 de junio 2020).

P1: Sí, totalmente, sobretodo en el área de procesos, puesto que durante mi carrera se me ha inculcado que lo más importante es generar procesos optimizados y entre ello entra la sostenibilidad (Participante 1, comunicación personal, 15 de junio 2020).

En relación con la percepción sobre la sostenibilidad en ingeniería, no existe un consenso común en el ámbito universitario, y el personal docente que trabaja la sostenibilidad a nivel curricular, presenta limitaciones, al evaluar las competencias asociadas con la sostenibilidad, tal como lo describe Albareda-Tiana y Gonzalo-Cirac (2013), quienes proponen un instrumento concreto para la evaluación de la sostenibilidad a nivel universitario. Otras personas investigadoras, como Antúnez et al., (2017), plantean que, en general, existe entre la comunidad universitaria un escaso conocimiento de los conceptos de sostenibilidad. Una manera de conocer la percepción de los miembros del GI acerca de la sostenibilidad en ingeniería, involucra indagar sobre las competencias relativas al conocimiento de los problemas de sostenibilidad, tal como menciona Rieckmann (2012) en su investigación. Sobre la sostenibilidad en ingeniería, las y los estudiantes opinan que es:

P4: Importante, puesto que los seres humanos debemos ser educados y preparados para la recuperación y preservación del medio ambiente. Solo una sociedad con una profunda y amplia formación tecnológica, humanista y renovable puede entender la importancia de la sostenibilidad (Participante 4, comunicación personal, 17 de junio 2020).

P5: Debido a la creciente necesidad, tanto de dar solución a problemáticas de índole ambiental como realizar proyectos de ingeniería amigables con el medio ambiente, la ingeniería sostenible es un enfoque que cada vez se hará más presente e imprescindible (Participante 5, comunicación personal, 18 de junio 2020).

P2: Es la ingeniería del futuro, todas las fases de cualquier proyecto deben enfocarse en ser ambientalmente sostenibles (Participante 2, comunicación personal, 15 de junio 2020).

Otras personas estudiantes destacan el papel de la sostenibilidad en la ingeniería del futuro y piensan que representa un cambio:

P8: Será la base en el futuro para la ingeniería, puesto que el uso óptimo de los recursos permitirá proveer de nuevos proyectos a las industrias, así como también a los sistemas de gobierno (Participante 8, comunicación personal, 19 de junio 2020).

P10: Estupenda, es absolutamente necesaria. El planeta está en constante cambio, y como ingenieros, debemos orientar ese cambio a su favor. (Participante 10, comunicación personal, 20 de junio 2020). 


\section{Relación entre sostenibilidad y trabajo colaborativo}

Después de analizar 43 documentos, relacionados con competencias en sostenibilidad (Wiek, Withycombe y Redman, 2011), estos generaron una competencia denominada competencia interpersonal, que es la habilidad para motivar, permitir y facilitar la colaboración y la participación en la investigación en sostenibilidad y en la resolución de problemas. Por su parte, Rieckmann (2012) nombra entre las competencias necesarias para el aprendizaje de EDS, la competencia para la cooperación en grupos heterogéneos y la competencia para el trabajo interdisciplinar. Al ser consultados sobre el uso de GI para la realización de futuros trabajos o aprendizajes que incluyan la sostenibilidad, las personas integrantes del GI expresaron:

P1: Lo recomendaría, sin dudarlo. En mi experiencia personal todos aprendimos, apoyamos y realizamos un buen trabajo. Los objetivos grupales fueron logrados, y la culminación del PEG fue todo un éxito gracias al trabajo en grupo interactivo (Participante 1, comunicación personal, 15 de junio 2020).

P2: Sí, en macro proyectos es necesario realizar grupos interactivos para el intercambio de ideas y experiencias, ya que estas pueden ayudar a la solución de dudas a lo largo del proyecto; siempre es bueno tener más de una opinión. Además, el intercambio de material es de mucha ayuda (Participante 2, comunicación personal, 15 de junio 2020).

Como reflexión final de la relación entre la ingeniería, GI y sostenibilidad opinaron:

P6: Considero que se debe apuntar hacia ella. El planeta cada día se queda con menos recursos para satisfacer nuestras necesidades, además de que nos encontramos en un alto grado de contaminación por cubrir las mismas; por lo que al guiar la ingeniería hacia el uso adecuado de estos recursos contribuye con que futuras generaciones puedan disfrutar de lo que hoy disfrutamos nosotros. Para ello es necesario tomar en cuenta grupos interdisciplinarios, de esta manera se pueden garantizar productos viables y económicos que reduzcan impactos ambientales negativos (Participante 6, comunicación personal, 18 de junio 2020).

\section{Discusión}

Los GI son una alternativa para construir aprendizajes significativos en las futuras personas profesionales. Los resultados de este trabajo coinciden con otros estudios (Díez-Palomar et al., 2010; Ponce, 2017; Prados y Aguilera, 2013; Flecha et al., 2014), en los cuales se reconoce el desarrollo de capacidades personales y profesionales que son imprescindibles para responder a las demandas sociales del mundo actual, entre ellas: el trabajo en equipo, la organización y gestión, la responsabilidad compartida, el respeto, la empatía, la solidaridad, entre otros.

$\mathrm{Al}$ respecto, Burns et al. (2019) plantean que crear una relación interactiva y dinámica entre la persona instructora y el alumnado, es un aspecto importante de la pedagogía de la sostenibilidad. De acuerdo con esta experiencia con GI, es posible plantear una línea de investigación para implementar la pedagogía de la sostenibilidad, según ejemplos como el de Tuzun (2020), quien estudia la sostenibilidad para ingenieras e ingenieros químicos en la Universidad de Cambridge, con el uso de la metodología del aprendizaje basado en problemas con grupos interactivos. Estas actividades logran la reflexión y la utilización constructiva del conocimiento en la población estudiantil de ingeniería.

Incluir la sostenibilidad dentro de la formación del profesorado y estudiantes de ingeniería de la UNET, permitirá a la población egresada de ingeniería a ser consciente de su entorno y tener una visión de desarrollo sostenible, que pueda aplicar en su desempeño como profesional. 


\section{Conclusiones}

Los hallazgos de este trabajo demuestran que la sostenibilidad se concreta en el trabajo colaborativo, y contribuye con la formación de profesionales de ingeniería que se comprometen con la protección de los recursos naturales. Las personas participantes estuvieron de acuerdo en reconocer que aprendieron acerca de la sostenibilidad mediante la participación en el GI, donde se valora la construcción de conocimientos y el desarrollo de competencias comunicativas y sociales, por cuanto exigió responsabilidad, tolerancia, respeto, entre otras habilidades necesarias para trabajar y aprender en conjunto.

Asimismo, existe la necesidad de promover el cuidado de la naturaleza en los y las estudiantes de ingeniería, así como el mejor aprovechamiento de los recursos naturales y la enseñanza a las futuras personas ingenieras sobre el conocimiento reflexivo y crítico del ambiente, es decir, de la relación existente entre el sistema social, cultural y natural de un contexto particular. Por tal razón se debe continuar en el estudio y desarrollo de este tipo de propuestas.

\section{RECOMENDACIONES}

De acuerdo con el análisis de esta experiencia, se debe incentivar un cambio en el proceso de formación docente en quienes se encargan de enseñar los principios de la sostenibilidad al estudiantado de ingeniería mediante la pedagogía ambiental y, de esta manera, incluir a ingenieros e ingenieras graduadas en Venezuela en la tendencia mundial de la conservación y aprovechamiento de recursos en todos los ámbitos de la ingeniería.

Por consiguiente, es necesaria la implementación de planes de desarrollo profesional para docentes, que incluyan prácticas pedagógicas bajo la corriente de la pedagogía de la sostenibilidad dentro del currículo de ingeniería de la UNET, como una manera de contribuir a los objetivos de desarrollo sostenible en Venezuela. De esta manera sería posible mejorar la calidad de la formación de docentes en las instituciones universitarias venezolanas, y promover la sostenibilidad en los programas de formación en ingeniería, para que al egresar las personas profesionales sean un agente transformador del ambiente, y lograr una visión integradora de los saberes construidos en su paso por la institución educativa.

\section{REFERENCIAS BIBLIOGRÁFICAS}

Albareda-Tiana, S., y Gonzalo-Cirac, M. (2013). Competencias genéricas en sostenibilidad en la educación superior. Revisión y compilación. Revista de Comunicación de la SEECI, 32, 141-159. doi: org/10.15198/ seeci.2013.32.141-159

Antunes, A., y Gadotti, M. (2005). La ecopedagogía como la pedagogía indicada para el proceso de la Carta de la Tierra. La Carta de la Tierra en acción. Amsterdam: Royal Tropical Institute.

Antúnez, M., Gomera, A., y Villamandos, F. (2017). Sostenibilidad y currículum: problemática y posibles soluciones en el contexto universitario español. Profesorado, 21(4), 197-214.

Aubert, A., Flecha, A., García, C., Flecha., R., y Racionero, S. (2010). Aprendizaje dialógico en la sociedad de la información (3era ed.). Barcelona: Hipatia Editorial, S.A.

Aznar, P., y Ull, M. (2009). La formación de competencias básicas para el desarrollo sostenible: el papel de la Universidad. Revista de Educación, 1, 219-237.

Bonilla-Castro, E., y Rodríguez, P. (2005). Más allá del dilema de los métodos. La investigación en Ciencias Sociales. Colombia: Editorial Norma.

Burns, H., Sybil, S y Spalding, H. (2019). Teaching Sustainability: Recommendations for Best Pedagogical Practices. Journal of Sustainability Education, 19, 1-16. 
Cheah, S., Yun, K., y Sale, D. (2012). Pedagogical approach to integrate sustainable development into engineering curriculum. En Queensland University of Technology, Proceedings of the 8th International CDIO Conference, (pp. 1-16). Brisbane, Australia.

Colás, P. (2000). Enfoques en la metodología cualitativa: sus prácticas de investigación. En L. Buendía, P. Colás, y F. Hernández (Eds.), Métodos de Investigación en Psicopedagogía (pp.225-249). Madrid, España: McGrawHill.

Comisión Europea (2015). Cerrar el círculo: un plan de acción de la UE para la economía circular. COM. Recuperado de https://bit.ly/3soZHku

Cristina, M. (2016). Promoting technological Entrepreneurship through Sustainable Engineering education. Procedia Technology, 22, 1129-1134. Doi: https://doi:org/10.1016/j.protcy.2016.01.159

Conferencia de Rectores de la Universidades españolas (CRUE). (junio, 2012). Directrices para la introducción de la Sostenibilidad en el Curriculum. Comité ejecutivo del Grupo de Trabajo de Calidad Ambiental y Desarrollo Sostenible. Recuperado de https://www.crue.org/wp-content/uploads/2020/02/Directrices_Sosteniblidad_C rue2012.pdf

Díaz, J., Juárez, G., García, F., Rosales, W., y Zambrano-Sandoval, H. (2018). Horno cerámico sustentable para productos artesanales de arcilla en Latinoamérica. Revista Cientifica UNET, 30(1), 80-90.

Díaz, J., Molina, J., y Prato, J. (2016). Energy-Environmental Diagnosis of the Ceramic Sector Companies in the Metropolitan Area of Cucuta, Norte de Santander, Colombia. Key Engineering Materials, 663, 133-139. doi: h ttps://www.scientific.net/KEM.663.133

Díez-Palomar, J., García, P., Molina, S., y Rué, L. (2010) Aprendizaje dialógico en las matemáticas y en las ciencias. Revista Interuniversitaria de Formación del Profesorado, 67(24), 75-88.

Downing, K., Kwong T., Chan, S., Lam, T., y Downing, W. (2009). Problem-based learning and the development of metacognition. Higher Education, 57, 609-621.doi: https://doi.org/10.1007/s10734-008-9165-x

Flecha, R., Racionero, S., Tintoré, M., y Arbós, A. (2014). Actuaciones de Éxito en la Universidad. Hacia la Excelencia Tomando las Mejores Universidades como Modelo. Multidisciplinary Journal of Educational Research, 4(2), 131-150. doi: https://doi.org/10.4471/remie.2014.08

Gómez, M., y Aguilera, A. (2015). Valoración de comunidades de aprendizaje por el voluntariado universitario. EA, Escuela abierta, Revista de Investigación Educativa, 18, 23-54. Recuperado de https://dialnet.unirioja.es/servle $\mathrm{t} /$ articulo? codigo $=5752658$

Gómez-del-Castillo, M., Aguilera-Jiménez, A., y Prados-Gallardo, M. (2016). El voluntariado de la Universidad de Sevilla en los grupos interactivos de las comunidades de aprendizaje. Profesorado, 20(2), 278-295. Recuperado de https://www.redalyc.org/articulo.oa?id=567/56746946015

Huntzinger, D., Hutchins, M., Gierke, J., y Sutherland, J. (2007). Enabling Sustainable Thinking in Undergraduate Engineering Education. International Journal of. Engineering Education, 23(2), 218-230.

Martínez, M (2006). Ciencia y arte en la metodología cualitativa. (2da. ed.) México: Trillas.

McDonough, W., y Braungart, M. (2005). De la Cuna a la Cuna [Cradle to Cradle]. Rediseñando la forma en que hacemos las cosas. Madrid: McGrawHill.

Ministerio del Medio Ambiente y Ministerio de Educación Nacional. (2002). Política Nacional de Educación Ambiental SINA. República de Colombia. Recuperado de https://bit.ly/3d6ADZ2

Murray, A., Skenne, K. y Haynes, K. (2015). The Circular Economy: An Interdisciplinary Exploration of the Concept and Aplication in a Global context. Journal of Business Ethics, 140, 369-380 doi: https:doi.org/10.1007/ s10551-015-2693-2.

Naciones Unidas. (2016). Agenda 2030 y los objetivos de desarrollo sostenible. CEPAL. Recuperado de https://bit.ly $13 \mathrm{jt} 0 \mathrm{dcf}$

Organización de las Naciones Unidas [ONU]. (1987). Report of the World Commission on Environment and Development: Our Common Future. [Informe Brundtland]. Oxford: Oxford University Press.

Painter, D. (2003). Forty-nine shades of green: ecology and sustainability in the academic formation of engineers. Ecological Engineering, 20(4), 267-273. doi: https://doi.org/10.1016/S0925-8574(03)00008-9 
Peirats, J. y López, M. (2013). Los grupos interactivos como estrategia didáctica en la atención a la diversidad. ENSAYOS, 28, 197-211. Recuperado de http://www.revista.uclm.es/index.php/ensayos

Pérez, G. (2000). Investigación Cualitativa. Retos e interrogantes. II Técnicas y análisis de datos. (3era ed). Madrid: La Muralla.

Ponce, M. (2017). Grupos interactivos en educación universitaria: Estrategia orientada al éxito para el aprendizaje con sentido. Opción, 33(84), 404-439.

Prados, M., y Aguilera, A. (2013). Los alumnos y alumnas de la Universidad de Sevilla como voluntarios en comunidades de aprendizaje. Andalucía Educativa Revista Digital, 79, 1-6. Recuperado de https://idus.us.es/h andle/11441/31140

Rieckmann, M. (2012). Future-oriented higher education: Which key competencies should be fostered through university teaching and learning?. Futures, 44, 127-135.

Sinakou, E., Boeve, J., Goossens, M., y Van Petegem, P. (2018). Academics in the field of education for sustainable development: Their conceptions of sustainable development. Journal of Cleaner Production, 184, 321-332.

The Ellen MacArthur Foundation (2017). Economia Circular. Recuperado de https://www.ellenmacarthurfoundati on.org/es/economia-circular/concepto

Tobón, S. (2005). Formación basada en competencias. Pensamiento complejo, diseño curricular y didáctica (2da. ed.). Bogotá, Colombia: Ecoe.

Tuzun, U. (2020). Introduction to systems engineering and sustainability part II: Interactive teaching of sustainability modeling by systems integration design for chemical and biological engineers. Education for Chemical Engineers, 31, 94-104. doi: https://doi.org/10.1016/j.ece.2020.04.005

UNESCO (2005). El Decenio de las Naciones Unidas para la EDS. UNESCO, Paris. Recuperado de https://bit.ly/ 3 cki7xa

Wiek, A., Withycombe, L., y Redman, C. (2011). Key competencies in sustainability: a reference framework for academic program development. Sustainability Science, 6(2), 203-218.doi: https://doi.org/10.1007/s1 1625-01 $1-0132-6$

\section{Anexo}

\section{Guion de preguntas}

Estimados participantes. El presente instrumento tiene como propósito conocer sus aprendizajes y experiencias sobre el desarrollo sostenible a través de los grupos interactivos implementados durante la realización de su proyecto especial de grado (PEG).

Parte I. Seguidamente encontrará una serie de interrogantes, por favor responda según su opinión.

$1_{¿}$ Describa su experiencia al pertenecer a un grupo interactivo durante la realización de su proyecto especial de grado (PEG)?

2. ¿Cuál es su percepción acerca de la sostenibilidad en ingeniería?

3. ¿Usted cree que durante su desempeño como ingeniero puede contribuir a la sostenibilidad?, explique su respuesta

4. Exprese su opinión en relación con el entorno de aprendizaje, para ello tenga en cuenta, aspectos como: comunicación, cooperación u otros que usted considere influyeron para llevar a cabo el trabajo propuesto y el PEG.

5. Describa en sus propias palabras como fue el proceso de aprendizaje durante la realización de su PEG

6:Qué aprendió de sus compañeros de experiencia en el grupo interactivo?

7. ¿Cuál es su opinión sobre la relación entre el uso de subproductos en procesos de ingeniería y la sostenibilidad? 
Parte 2. En las siguientes interrogantes, marque aquellas que más se aproximen a su opinión.

¿Qué aprendió de la experiencia de trabajo como grupo interactivo durante realización de su PEG?

a) Desarrollar operaciones cognitivas elementales, tales como la memoria, la observación, el análisis y la síntesis

b) Aprender las reglas prácticas para la ejecución de un dominio de tareas

c) Elaborar y utilizar mecanismos de pensamiento para la resolución de problemas

d) Desarrollar procesos de aprendizaje cooperativo

e) Utilizar en el grupo interactivo todas las interacciones necesarias para afrontar exitosamente el PEG en la actual sociedad de la información

f) Interactuar con sus compañeros para conseguir objetivos comunes

g) Otro:

9. ¿Cuáles fueron las ventajas del trabajo cooperativo para la realización de su PEG?

a) Se comparte el proceso de enseñanza y aprendizaje

b) Se presenta interdependencia positiva, que contribuye a mejorar las relaciones entre iguales

c) Mejora la autoestima del estudiantado y promueve a que se establezcan relaciones positivas entre iguales

d) Incremento del aprendizaje instrumental y la motivación del estudiantado

e) Distribución del trabajo en función del tiempo y disponibilidad de equipos

f) Otro:

10. ¿Cuáles fueron las desventajas del trabajo cooperativo para la realización de su PEG?

a. Problemas para compartir los materiales y contenidos, además de la dificultad de relacionarlos falta de práctica para trabajar cooperativamente algunos de los y las estudiantes no tenían interés y delegaban el trabajo en el resto del grupo

d. Es necesaria una buena coordinación para trabajar en equipo y se necesita mucho tiempo para preparar las actividades de esta manera

e. Otro:

11. ¿Recomendaría el uso de Grupos Interactivos para la realización de futuros trabajos o aprendizaje?, justifique su respuesta.

\section{INFORMACIÓN ADICIONAL}

Cómo citar: Díaz García, J.I. y Chacón-Corzo, M.A. (2021). Los Grupos Interactivos en la formación para la sostenibilidad del estudiantado de ingeniería. Revista Educación, 45(2). Recuperado de http://doi.org/1 $0.15517 /$ revedu.v45i1.43541 